Objective This aimed to identify the determinants of prolonged length of stay in the hospital among victims of road traffic injury.

Methods A retrospective cohort study was conducted. Sociodemographics, accident details (e.g. time of injury, type of victim, other party to the accident, time to admission, intoxication, and helmet use), the Glasgow Coma Score upon admission, head injuries, torso injuries, injuries to the limbs, and length of stay) were abstracted from the patient charts and cleaned accordingly. The predictor model for the determinants of prolonged length of stay in the hospital was built.

Results There were 427 road traffic injury victims that were admitted during the time period considered. The distribution of the time to admission differed between those who have a short length of stay and long stay differed significantly $(p<0.01)$. Glasgow coma score, type of victim, mortality, age, and sex were found to be important determinants of prolonged hospital stay. Those who have a low Glasgow Coma Score (i.e. more severe cases) were found to be 2.77 (95\% CI: 1.13-6.91) times more likely to stay longer in the hospital as compared to those who have high Score. There is strong evidence for this association. Passengers are 78\% (95\% CI: 0.06-0.84) less likely to stay longer in the Hospital as compared to drivers. There is also strong evidence for this association.

Conclusion Based on the predictive model from 427 traffic injury patients, determinants of prolonged hospital stay were identified. Consequent policies and programs should address this.

\section{P.3.21 PREDICTORS OF HEALTH PERCEPTION AMONG WASTE PICKERS IN LANDFILL SITES IN JOHANNESBURG MUNICIPALITY, SOUTH AFRICA}

\footnotetext{
1,2 Felix Made*, 'Vusi Ntlebi, ${ }^{1}$ Tahira Kootbodien, ${ }^{1,2}$ Tlotleng Nonhlanhla, 1,2Kerry Wilson, 3,2 Ndaba Mpume, ${ }^{3,2}$ Kgalamono Spo, 1,2,4 Nisha Naicker. 'Epidemiology and Surveillance Section, National Institute for Occupational Health, National Health Laboratory Service, Johannesburg, South Africa; ${ }^{2}$ University of Witwatersrand, School of Public Health, Faculty of Health Sciences, Johannesburg, South Africa; ${ }^{3}$ Occupational Medicine Section, National Institute for Occupational Health, National Health Laboratory Service, Johannesburg, South Africa; ${ }^{4}$ Environmental Health Department, Faculty of Health Sciences, University of Johannesburg, Johannesburg 2028, Johannesburg, South Africa
}

\subsection{6/OEM-2019-EPI.280}

Background In South Africa, waste is disposed in landfill sites where waste pickers collect recyclable waste. These informal workers are exposed to various health hazards. However, seeking health care is often determined by the individual's perception of the status of their health. The objective of this study was to find predictors of health perceptions among waste pickers in Johannesburg Municipality, South Africa.

Methods A cross sectional study was conducted among waste pickers aged 18 years or older, working in major landfill sites in Johannesburg. Information on possible risk factors for health perception were collected. Health perception was defined as very good, good, fair, and poor. Ordinal logistic regression model with landfill sites taken as a cluster variable was done to find factors associated with health perception.

Results A total of 361 participants were included in this study. More than $70 \%$ of them were males. Their median age was 31 years (interquartile range (IQR): 27-39). Ever smoked (OR: 1.72; 95\% CI: 1.112-2.665), mental ill health risk (OR: 1.87; 95\% CI: 2.849), chronic diseases (OR: 2.34; 95\% CI:1.479-3.689), and history of infectious diseases (OR: 2.07; 95\% CI: 1.779-3.635) were all statistically significantly associated with increased odds of reporting poor health. Participants who reported an injury were less likely to report poor health (OR: 0.53; 95\% CI: 0.307-0.911).

Conclusions Predictors for reporting poor health among waste pickers were smoking, chronic and infectious diseases, and mental health risk. However, injuries from cuts showed a decreased odd of reporting poor health. Mitigating these risk factors including health promotion as well as improving primary health care may improve perceptions of good health among waste pickers in SA.

\section{P.3.23 INCIDENCE RATE AND RELATED FACTORS OF OCCUPATIONAL INJURIES AMONG VETERINARIANS}

${ }^{1}$ Napattamon Manorat*, ${ }^{2}$ Soontorn Supapong. ${ }^{1}$ Roi Et Hospital, Roi Et, Thailand; ${ }^{2}$ Department of Preventive and Social Medicine, Chulalongkorn University, Bangkok, Thailand

\subsection{6/OEM-2019-EPI.281}

This cross-sectional descriptive study was conducted to determine the incidence rate and patterns of occupational injuries and to determine the factors which related to occupational injuries among veterinarians in veterinary clinics in Bangkok. The self-administered questionnaires were distributed to 395 veterinarians who had more than one-year experience in each veterinary clinic. Data were analyzed using Chi-square test, Fisher's Exact test and Independent t-test.

The results found that Incidence rate of occupational injuries among these veterinarians were 280 events per million man-hours and 56.22 times per 100 full-time veterinarians. The most site of injury was hand. Abrasion and laceration were the most common injuries. Dogs and cats bite were the most causes of injuries. Diagnostic process was the major activity associated with injury. Sixty-nine percent of this sample required physician treatment and fiftysix percent needed work restriction. Thus, the mean of work days lost was 3.45 days. Factors related with occupational injuries were age ( $p$-value $<0.001)$, year of graduation $(\mathrm{p}$-value $=0.003)$, BMI $(\mathrm{p}$-value $<0.001)$, allergic problem $(\mathrm{p}$ value $=0.001)$, sleep less than 7 hours $(p$-value $=0.022)$, drowsy pills $(p$-value $=0.003)$, exercise $(p$-value $=0.024)$, type of practice $(\mathrm{p}$-value $<0.001)$, year in practice ( $\mathrm{p}$ value $<0.001)$, working time more than 40 hours per week $(p$-value $=0.035)$, safety training required $(p$-value $=0.038)$, careful animal restraint ( $\mathrm{p}$-value $=0.036)$, always using safety bin $\quad(p$-value $=0.01), \quad$ adequate assistants $\quad(p$-value $=0.01)$, adequate animal restrained equipment ( $p$-value $<0.001)$, and adequate ambient light ( $\mathrm{p}$-value $=0.018)$. According to the results from this research, the incidence rate of occupational injury among veterinarians could be reduced by appropriated adjustment of working and resting period, increasing awareness and knowledge transfer experience. Moreover, working area should be properly prepared. 